\title{
Evaluación experimental de la eficiencia de una pintura repelente para arañas del género Loxosceles
}

\author{
Alejandro Catalán ${ }^{1 a}$, Jorge E Araya2a, Héctor Varela ${ }^{3 b}$, \\ William Cortés ${ }^{1 a}$, Hernán Sagua ${ }^{2 a}$, Jorge G onzález ${ }^{2 a}$. \\ against the spider Loxosceles laeta
}

Background: Loxoscelism is a severe reaction to the bite of the spider Loxosceles laeta. In recent years, a paint with repellent properties has been promoted in the commerce. However, there are no reports of experiments evaluating its effectiveness. Aim: To evaluate experimentally the repellent properties of a paint against Loxosceles laeta. Material and methods: Males, females and nymphs of L laeta were deposited in cockpits that allow the free displacement of the spider. Half of the cockpit was covered with repellent paint. Daily observations during one week, determined how frequently the spiders occupied the space covered with repellent paint. The experiments were run in triplicate. Results: No statistical differences in the occupancy of spaces covered with repellent paint or not covered with it were observed for nymphs ( $87 \%$ and $67 \%$, respectively), males ( $72 \%$ and $77 \%$, respectively) or females (91\% and $84 \%$, respectively). Conclusions: The tested paint does not have a repellent action against the spider Loxosceles laeta (Rev Méd Chile 2009; 137: 240-5).

(Key words: Insect repellents; Loxosceles venom; Spider venoms)

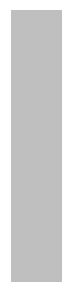

\footnotetext{
Recibido el 10 de enero, 2008. Aceptado el 10 de noviembre, 2008. Trabajo financiado por proyecto FONDEF D04I1247.

${ }^{1}$ Unidad de Parasitología Molecular, Universidad de Antofagasta, Chile. ${ }^{2}$ Unidad de Parasitología, Departamento de Tecnología Médica, Facultad Ciencias de la Salud, Universidad de Antofagasta, Chile. ${ }^{3}$ Departamento de Matemáticas, Universidad de Antofagasta, Chile.

Tecnólogo Médico

boctor en Ciencias Matemáticas
}

L oxosceles laeta, conocida como "araña de los _rincones" es un arácnido de hábitos nocturnos e intradomiciliarios. Su cuerpo mide $10-15 \mathrm{~mm}$ de largo y $42-45 \mathrm{~mm}$ con las patas extendidas y su

Correspondencia a: Dr. Jorge González Cortés. Av. Universidad de Antofagasta 02800. Fax: 55-637802.

E mail: jgonzalez@uantof.cl abdomen es más oscuro que el resto del cuerpo y extremidades. Se establece en entretechos, rincones de habitaciones, detrás de cuadros, en closets, estantes, en la cara inferior de muebles y en ocasiones en prendas de vestir que han estado colgadas en las paredes. En estos sitios teje una tela en la cual deposita sus huevos en capullos que ella construye ${ }^{1,2}$. Llaeta es una araña solitaria que vive y caza aislada de sus congéneres, es 
huraña y rehuye la presencia del hombre. No obstante, durante las noches abandona su tela y camina por las paredes en busca de insectos. Cuando su tránsito es interrumpido por la presencia de una cama apegada a la pared o decide refugiarse en prendas de vestir colgadas en la pared, se generan condiciones para el encuentro accidental con el hombre, donde el arácnido al sentirse atacado responde mordiendo mediante dos potentes quelíceros e inoculando su veneno. Este accidente que habitualmente ocurre durante el sueño nocturno o en las primeras horas de la mañana se denomina loxoscelismo ${ }^{1,3}$ y puede evolucionar como un cuadro de intoxicación bajo dos formas clínicas, denominadas loxoscelismo cutáneo (LC) y loxoscelismo cutáneo-visceral (LCV) o sistémico ${ }^{4,5}$.

En los Servicios de Urgencia de la ciudad de Antofagasta, la mordedura por araña de los rincones es motivo frecuente de consulta (alrededor de 70 ingresos semestrales por cuadros de loxoscelismo) al Servicio de Medicina del Hospital Clínico Regional. En virtud de lo anterior, la implementación sistemática de medidas orientadas a prevenir la mordedura accidental por Llaeta constituye una real necesidad.

Dentro de las estrategias de profilaxis, se encuentran aquellas tendientes a reducir las posibilidades de encuentro entre el hombre y el arácnido. La utilidad de estas medidas incluye el aseo riguroso de la vivienda humana, el evitar colgar prendas de vestir en las paredes y el no apegar las camas a las paredes, entre otras. Por otro lado, el uso de substancias repelentes ha sido sistemáticamente utilizado en la profilaxis de la picadura y mordedura de artrópodos ${ }^{6}$. Sin embargo, poco se conoce acerca del uso de substancias repelentes contra arácnidos. De esta manera, la reciente introducción al mercado de una pintura con propiedades repelentes contra la araña de rincón, podría ofrecer una opción en la perspectiva de una nueva forma de profilaxis del cuadro tóxico producido por mordedura de arañas del género Loxosceles.

En esta comunicación, se presenta un estudio en el cual se evaluó experimentalmente la efectividad de una pintura con actividad repelente contra la araña de rincón, mediante un diseño experimental que simula en un microambiente aislado, el efecto que tendría la aplicación de esta pintura en muros y techos de un domicilio real.

\section{Material y MÉTODO}

Recolección de ejemplares L laeta en domicilios de Antofagasta. Un total de 90 ejemplares de L laeta fueron capturados en diferentes ambientes intradomiciliarios de la ciudad de Antofagasta. Los ejemplares fueron transportados al laboratorio de Parasitología Molecular de la Facultad de Ciencias de la Salud de la Universidad de Antofagasta y mantenidos en recipientes plásticos herméticos por un periodo de tiempo no mayor de 7 días para comprobar su vitalidad hasta el momento de su utilización. Los arácnidos una vez identificados según características morfológicas 7,8 y diferenciados según estadio y sexo, fueron divididos en tres grupos compuestos cada uno por 30 ejemplares: hembras, machos y ninfas, respectivamente. Todos los estadios fueron mantenidos en habitación a temperatura ambiente en oscuridad, hasta el momento de su utilización.

Evaluación experimental de una pintura antiarañas de rincón. Para la evaluación experimental del efecto repelente de la pintura anti-araña de los rincones, se empleó una metodología en la cual se diseñaron y fabricaron diez cajas de madera con cubierta de melamina (Figura 1). Este tipo de material se escogió por su bajo costo y su menor capacidad de absorción. Las cajas fueron preparadas para contener en su interior a un espécimen del arácnido. Cada caja proporcionaba dos habitáculos con ambientes separados por un tabique con orificio que permitía el libre tránsito de la araña desde un ambiente a otro. Cinco cajas fueron utilizadas como control (C 1-5) y en ellas el primer habitáculo se encontraba recubierto con 2 a 3 capas de pintura látex no repelente (mínimo de capas recomendado por el fabricante), tanto en sus muros como en sus caras superiores, mientras que el segundo habitáculo no se encontraba pintado y sus caras superiores estaban cubiertas con la misma madera para evitar el paso de la luz. Otras 5 cajas fueron utilizadas como experimentales (E 1-5) y en ellas uno de los habitáculos se encontraba recubierto con 2 a 3 capas de pintura látex anti-araña de rincón y el segundo ambiente no se encontraba pintado.

En todos los casos, la superficie, porosidad y textura de los ambientes revestidos con pintura no fueron diferentes de aquellos que los arácnidos 


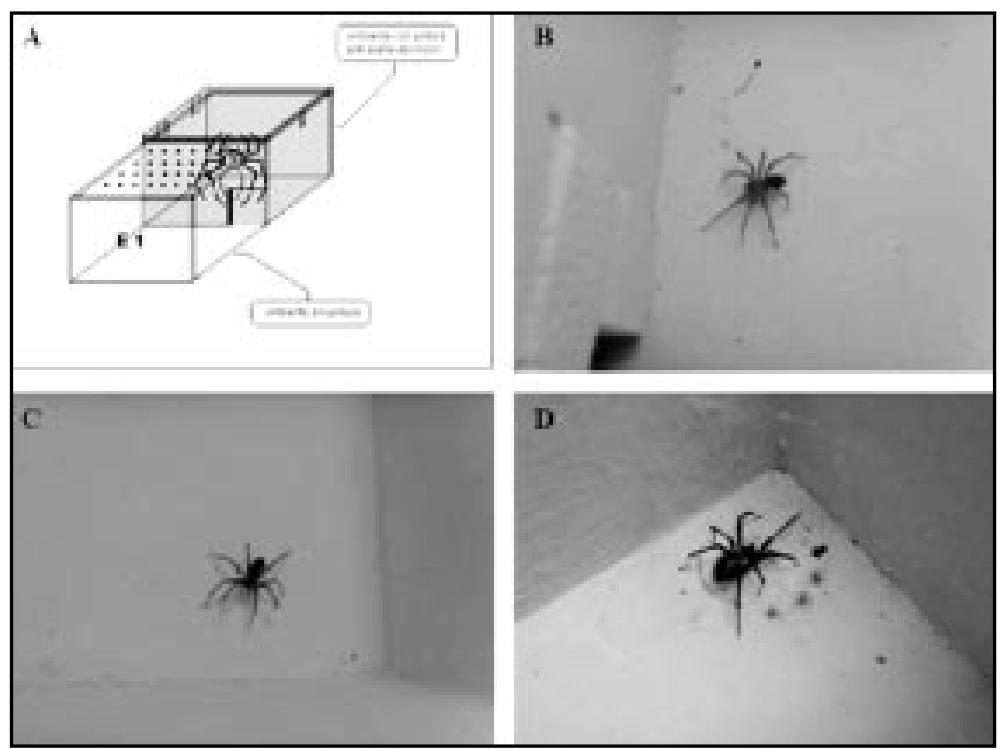

Figura 1. A) Esquema del habitáculo utilizado para evaluar en condiciones de laboratorio el efecto repelente de la pintura anti-arañas de rincón. B) Establecimiento del arácnido en el ambiente con pintura anti-arañas de rincón. C) Arácnido en ambiente con pintura sin repelente. D) Presencia de ooteca en los grupos de hembras de Llaeta usadas para evaluar el efecto de la pintura anti-arañas de rincón, donde la ooteca está indicada con una flecha.

podrían encontrar en un muro convencional. La aplicación de la pintura se realizó un día antes de iniciado el experimento y tanto la pintura repelente como la no repelente fueron de la misma marca, color y calidad. Ambas series experimentales se mantuvieron en idénticas condiciones de temperatura y humedad en habitaciones separadas. Se registró el habitáculo de permanencia del arácnido diariamente en el mismo horario durante 7 días. Cada experimento se realizó en triplicado y las observaciones fueron realizadas por una misma persona.

Estadística. Los datos obtenidos de E (1-5) y C (1-5), para cada serie de estudio, según estadios (ninfas, hembras y machos), fueron tabulados y sometidos a análisis estadístico basados en pruebas Chi-cuadrado mediante software Statgraphics Plus 5.1 y la prueba exacta de Fisher, para un tamaño de muestra pequeño $(n=5)$, a un nivel de significación de 0,05 .

\section{Resultados}

Efecto de la pintura anti-araña de rincón sobre L laeta. En relación a la serie de hembras, se registró que en 91,4\% de las observaciones, las hembras permanecieron en el habitáculo con pintura antiaraña de rincón, mientras que entre las hembras del grupo control se registró 84,29\% de especímenes que permanecían en el espacio con pintura sin repelente (Figura 2). En la serie machos, se registró que en $72,85 \%$ de las observaciones, éstos se encontraron habitando el habitáculo con pintura anti-araña de rincón, mientras que entre los del grupo control se observó una permanencia de 77,14\%, (Figura 2). Finalmente, en la serie correspondiente a ninfas se registró $87,15 \%$ de permanencia en el habitáculo con pintura anti-araña de rincón, mientras que entre las ninfas del grupo control este parámetro fue de $67,15 \%$ de permanencia en el habitáculo con pintura sin repelente (Figura 2).

Colonización de L laeta en el ambiente con pintura anti-araña de rincón. Paralelo a la evaluación del efecto repelente de la pintura anti-araña de rincón sobre los diferentes estadios de L laeta, se realizaron observaciones para evaluar si esta pintura era además capaz de afectar la colonización de su nuevo hábitat experimental por parte 


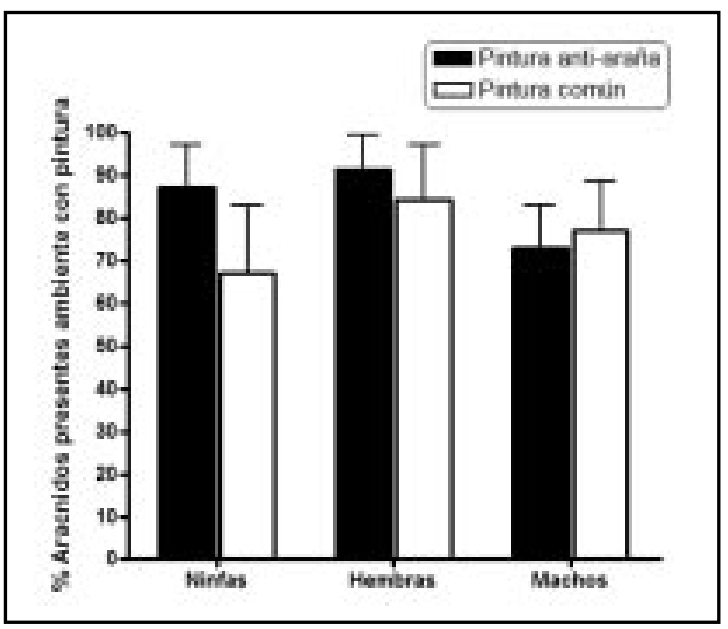

Figura 2. Efecto de la pintura anti-araña de los rincones sobre los diferentes estadios de L laeta. Los experimentos se realizaron en triplicado y son representativos de experimentos independientes, ejecutados con idéntico protocolo y similares resultados.

de la araña. Como se observa en la Figura 1D y Tabla 1, 11 de las 30 hembras evaluadas $(36,6 \%)$ elaboraron ooteca sobre la superficie recubierta con pintura repelente, mientras que $17(56,6 \%)$ también lo hicieron dentro de las arañas del grupo control.

Estadística. En este caso, la hipótesis de nulidad establece que la proporción de L laeta presentes en los habitáculos con pintura no repelente es igual a la proporción de L laeta presentes en los habitáculos tratados con látex anti-arañas de rincón. Valores del estadístico exacto de Fisher indican para todos los grupos en estudio el no efecto de la pintura sobre L laeta, donde no se rechaza la hipótesis de nulidad y se descarta la hipótesis alternativa de una mayor proporción de Llaeta en habitáculos tratados con la pintura látex anti-araña de rincón respecto de los arácnidos presentes en los habitáculos sin pintura antiarañas, donde no existe efecto repelente. Los tests estadísticos para evaluar asociación entre la pintura y el estadio evolutivo de L laeta, indicaron que no existe asociación entre la presencia de la pintura y el estadio evolutivo de L laeta en estudio.

Tabla 1. Porcentaje de hembras de L laeta con presencia de ooteca

\begin{tabular}{|lcr|}
\hline Hembras L laeta & $\mathbf{N}^{\circ}$ & $\%$ \\
\hline En habitáculo con pintura repelente & & 36,6 \\
Presencia de ooteca & 11 & 63,4 \\
Sin presencia de ooteca & 19 & \\
& & 56,6 \\
En habitáculo con pintura control & 17 & 43,4 \\
Presencia de ooteca & 13 & 100,0 \\
Sin presencia de ooteca & 30 & \\
Total & & \\
\hline
\end{tabular}


DisCUSIÓN

El loxoscelismo, en sus diferentes formas clínicas, es un accidente eminentemente domiciliario, que ocurre debido a factores absolutamente evitables. Por ello, es necesario evaluar las medidas de vigilancia y control que las personas adoptan para evitar o reducir el riesgo de la mordedura de araña, especialmente las nuevas propuestas de control, como es el uso de pintura repelente. Lo anterior, resulta especialmente relevante en la ciudad de Antofagasta, debido a la alta casuística observada y la presentación de cuadros de envenenamiento severo (LCV) con desenlace fatal que con cierta frecuencia se reportan en el Servicio de Medicina del Hospital Clínico Regional de Antofagasta $^{9,10}$.

Componentes con actividad repelente contra artrópodos, principalmente derivados de plantas, han sido sistemáticamente empleados contra mosquitos y garrapatas. Entre estos compuestos destacan el N, N-dietil-3-metilbenzamida (DEET), permetrina, picaridina, aceite de citronella y el aceite de eucaliptus limón ${ }^{6}$. En general, los compuestos usados como substancias repelentes deben poseer como características un efecto prolongado, ser atóxicos para el ser humano, poseer un bajo costo y ser inodoros o carentes de olor desagradable ${ }^{13}$. De igual manera, el uso de pinturas insecticidas ha sido propuesto como una estrategia de control de triatominos ${ }^{11,12}$. Sin embargo, con excepción de los repelentes de uso personal que se aplican en ropa o directamente sobre la piel, el uso de pinturas repelentes o insecticidas no ha tenido aplicación a gran escala. En el caso particular de la pintura insecticida contra triatomas, aunque la literatura reporta un relativo buen efecto, éstas no llegaron a ser comercializadas al observarse problemas en su estabilidad y viscosidad ${ }^{12}$.

No obstante, no existen en la literatura científica reportes respecto al uso de pinturas repelentes contra arácnidos de hábitat domiciliario. En virtud de lo anterior y considerando la introducción en el mercado nacional de una pintura repelente de arañas de rincón, evaluamos su efecto en el comportamiento de machos, hembras y ninfas de L laeta, que en nuestro país es la especie de arácnidos domiciliarios más prevalente y peligrosa. Para este efecto, se utilizaron habitáculos con ambientes recubiertos con pintura látex repelente en el grupo experimental y ambientes recubiertos con pintura látex del mismo color y calidad sin efecto repelente en los controles. De esta manera, pudimos observar que todos los estadios estudiados fueron poco sensibles al efecto de esta pintura. Más aún, en los experimentos realizados con las hembras de L laeta, se observó que además de permanecer en una alta proporción (91,4\%), en el ambiente con pintura-anti araña de rincón, 36,6\% de ellas fue capaz de desarrollar allí nido y ooteca, lo que es indicativo de una condición de asentamiento y adaptación del artrópodo a un nuevo ambiente (Figura 1D, Tabla 1).

Del mismo modo, los estadios ninfales, considerados teóricamente más susceptibles al efecto que podría causarles la pintura, mostraron una frecuencia de permanencia en el habitáculo recubierto con pintura repelente, similar a la observada en el grupo de hembras $(87,15 \%)$. Los resultados mostrados por las series experimentales fueron muy similares a los observados en las series del grupo control (Figura 2). De esta manera, la frecuencia con que los arácnidos permanecieron sobre la superficie cubierta con pintura repelente versus los que permanecieron sobre la pintura normal no repelente (pintura normal) no mostraron diferencias estadísticamente significativas $(\mathrm{p}>0,05)$.

El uso de cajas o habitáculos en la evaluación experimental del efecto de insecticidas y substancias repelentes, es el procedimiento habitualmente aceptado para este tipo de estudios. De modo general, consiste en disponer un determinado número de ejemplares en cada habitáculo, en el que previamente o con posterioridad se aplica la substancia a evaluar ${ }^{13,14}$. No obstante en nuestro estudio, por tratarse de una especie de hábitos solitarios, que defiende su territorio y que practica el canibalismo, se debió disponer de cada ejemplar en habitáculos separados.

De esta manera, es posible concluir, que al menos en las condiciones evaluadas en nuestro laboratorio, la pintura repelente no tiene efecto sobre los tres estadios de Llaeta. La composición y el principio repelente de esta pintura, por tratarse de un producto comercial protegido por patente, no es conocida. Sin embargo, es posible que este principio repelente sea inestable o posea una vida media corta que pudiese ser afectado por 
diferentes condiciones de almacenamiento o que pudiesen existir diferencias en distintos lotes de fabricación. Por lo anterior parece también necesario evaluar pinturas provenientes de diferentes lotes de fabricación. De igual manera, diferencias genéticas entre subpoblaciones de L laeta, que resulten en ejemplares con una menor susceptibilidad al efecto repelente no pueden ser descartadas.

A la luz de estos resultados, y en ausencia de otras alternativas, la práctica permanente de medidas tales como el aseo riguroso de la vivienda humana usando especialmente la aspiradora en

\section{REFERENCIAS}

1. Schenone H. A propósito del loxoscelismo en Chile. Rev Méd Chile 2004; 132: 121-2.

2. Schenone H, SaAvedra T, Rojas A, Villarroel F. Loxoscelismo en Chile. Estudios epidemiológicos, clínicos y experimentales. Rev Inst Med Trop S Paulo 1989; 31: 403-15.

3. Atias A. Parasitología Médica. Ed. Mediterráneo $1^{\underline{a}}$ Edición Santiago de Chile. 1999; 496-502.

4. Schenone H, Suárez G. Venoms of Scytodidae. Genus Loxosceles. A. Distribution and biology of Venenous Species; Chemistry, Toxicity, Pharmacology, and Mode of Action of Venom. B. Epidemiology, Symptomatology, Pathology, Prognosis, Treatment, and Prevention of Envenomations. En: Bettini S. Ed. Arthropod Venoms. Springer-Verlag Berlin Heidelberg New York. 1978; 247-75.

5. Schenone H. Toxic pictures produced spiders bites in Chile: latrodectism and loxoscelism. Rev Med Chil 2003; 131: 437-44

6. Katz TM, Miller JH, Hebert AA. Insect repellents: historical perspectives and new developments. J Am Acad Dermatol 2008; 58: 865-71.

7. Aguilera Ma, Casanueva ME. Arañas chilenas: estado actual del conocimiento y clave para las familias de araneomorphae. Gayana 2005; 69: 201-24. todos los rincones y espacios inaccesibles, sumado a evitar apegar la cama a la pared y colgar ropas en las paredes, siguen teniendo permanente vigencia como acciones de vigilancia y control frente a los accidentes provocados por la mordedura de araña de rincón. No obstante, resulta aún más necesario el implantar programas de educación sanitaria de la población, especialmente en la ciudad de Antofagasta en que la prevalencia domiciliaria de L laeta es alta. Finalmente, la búsqueda de nuevos compuestos activos con efecto repelente contra Llaeta parece ser altamente necesaria.
8. Gertsch WJ. The spider genus Loxosceles in South America (Araneae Scytodidae). Bull Am Mus Nat Hist 1967; 136: 117-74.

9. GutiérRez J, Sagua H. Loxoscelismo en el niño. Análisis de 15 casos. Hospital Regional Dr. Leonardo Guzmán (1973-1978) Antofagasta-Chile. Rev Chil Pediatr 1979; 50: 21-7.

10. Zambrano A, González J, Callejas G. Desenlace fatal por loxoscelismo cutáneo visceral. Rev Méd Chile 2005; 133: 219-23.

11. Avila Montes Ga, Ponce C, Ponce E, Martínez Hernández M, Flores M. Insecticidal paint and fumigant canisters for Chagas' disease control: community acceptance in Honduras. Rev Panam Sal Publ 1999; 6: 311-20.

12. Dias JC, Jemmio A. About an insecticidal paint for controlling Triatoma infestans, in Bolivia. Rev Soc Bras Med Trop 2008; 41: 79-81.

13. Bunner BL, Perich MJ, Boobar LR. Culicidae (Diptera) mortality resulting from insecticide aerosols compared with mortality from droplets on sentinel cages. J Med Entomol 1989; 26: 222-5.

14. Chung YK, Lam-Phua SG, Chua YT, Yatiman R. Evaluation of biological and chemical insecticide mixture against Aedes aegypti larvae and adults by thermal fogging in Singapore. Med Vet Entomol 2001; 15: 321-7. 